This article was downloaded by: [B-on Consortium - 2007]

On: 7 November 2008

Access details: Access Details: [subscription number 778384761]

Publisher Taylor \& Francis

Informa Ltd Registered in England and Wales Registered Number: 1072954 Registered office: Mortimer House, 37-41 Mortimer Street, London W1T 3JH, UK

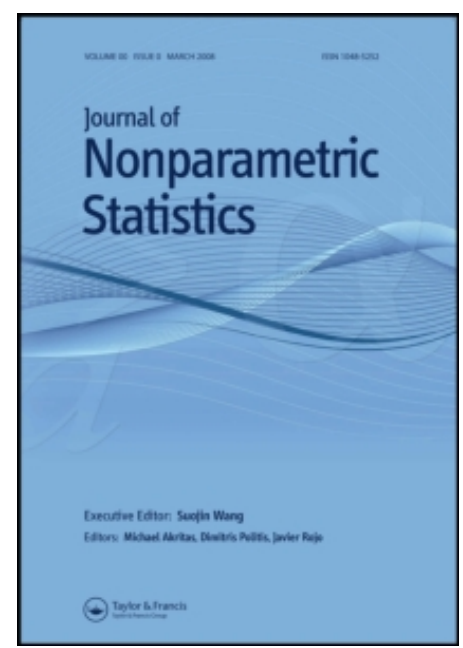

Journal of Nonparametric Statistics

Publication details, including instructions for authors and subscription information:

http://www.informaworld.com/smpp/title content=t713645758

\title{
Convergence rates for the estimation of two-dimensional distribution functions under association and estimation of the covariance of the limit empirical \\ process
}

Carla Henriques a; Paulo Eduardo Oliveira ${ }^{b}$

a Departamento de Matemática, Escola Superior de Tecnologia de Viseu, Campus Politécnico, Viseu,

Portugal ${ }^{b}$ Departamento de Matemática, Universidade de Coimbra, Apartado 3008, Coimbra, Portugal

Online Publication Date: 01 February 2006

To cite this Article Henriques, Carla and Oliveira, Paulo Eduardo(2006)'Convergence rates for the estimation of two-dimensional distribution functions under association and estimation of the covariance of the limit empirical process',Journal of Nonparametric Statistics, 18:2,119-128

To link to this Article: DOI: $10.1080 / 10485250500466119$

URL: http://dx.doi.org/10.1080/10485250500466119

\section{PLEASE SCROLL DOWN FOR ARTICLE}

\footnotetext{
Full terms and conditions of use: http://www.informaworld.com/terms-and-conditions-of-access.pdf

This article may be used for research, teaching and private study purposes. Any substantial or systematic reproduction, re-distribution, re-selling, loan or sub-licensing, systematic supply or distribution in any form to anyone is expressly forbidden.

The publisher does not give any warranty express or implied or make any representation that the contents will be complete or accurate or up to date. The accuracy of any instructions, formulae and drug doses should be independently verified with primary sources. The publisher shall not be liable for any loss, actions, claims, proceedings, demand or costs or damages whatsoever or howsoever caused arising directly or indirectly in connection with or arising out of the use of this material.
} 


\title{
Convergence rates for the estimation of two-dimensional distribution functions under association and estimation of the covariance of the limit empirical process
}

\author{
CARLA HENRIQUES $* \dagger$ and PAULO EDUARDO OLIVEIRA $\ddagger$ \\ †Departamento de Matemática, Escola Superior de Tecnologia de Viseu, Campus Politécnico, \\ 3504-510 Viseu, Portugal \\ \$Departamento de Matemática, Universidade de Coimbra, Apartado 3008, 3001-454 Coimbra, Portugal
}

(Received June 2002; revised in final form October 2005)

\begin{abstract}
Let $X_{n}, n \geq 1$, be an associated and strictly stationary sequence of random variables, having marginal distribution function $F$. The limit in distribution of the empirical process, when it exists, is a centred Gaussian process with covariance function depending on terms of the form $\varphi_{k}(s, t)=P\left(X_{1} \leq s, X_{k+1} \leq t\right)-F(s) F(t)$. We prove the almost sure consistency for the histogram to estimate each $\varphi_{k}$ and also to estimate the covariance function of the limit empirical process, identifying, for both, uniform almost sure convergence rates. The convergence rates depend on a suitable version of an exponential inequality. The rates obtained, assuming the covariances to decrease geometrically, are of order $n^{-1 / 3} \log ^{2 / 3} n$ for the estimator of $\varphi_{k}$ and of order $n^{-1 / 3} \log ^{5 / 3} n$ for the estimator of the covariance function.
\end{abstract}

Keywords: Association; Empirical process; Histogram estimator; Stationarity

AMS Classification: $62 \mathrm{G} 20 ; 62 \mathrm{G} 05 ; 62 \mathrm{G} 30$

\section{Introduction, assumptions and definitions}

Let $X_{n}, n \geq 1$, be a strictly stationary sequence of real-valued random variables with common continuous distribution function $F$. The empirical process induced by the sequence $X_{n}, n \geq 1$, is defined by $Z_{n}(t)=(1 / \sqrt{n}) \sum_{i=1}^{n}\left(I_{(-\infty, t]}\left(X_{i}\right)-F(t)\right)$, where $I_{A}$ represents the indicator function of the set $A$. The limit behaviour of the empirical process has been intensively studied because of its importance in many statistical applications. The limit in distribution of $Z_{n}(t)$ is well known to be a centred Gaussian process with covariance function $\Gamma(s, t)=F(s \wedge t)-F(s) F(t)$, if the variables are independent, or given by

$$
\Gamma(s, t)=F(s \wedge t)-F(s) F(t)+\sum_{k=1}^{\infty}\left(\varphi_{k}(s, t)+\varphi_{k}(t, s)\right),
$$

\footnotetext{
*Corresponding author. Email: carlahenriq@mat.estv.ipv.pt
} 
where $\varphi_{k}(s, t)=P\left(X_{1} \leq s, X_{k+1} \leq t\right)-F(s) F(t)$, when they are dependent. For practical purposes, we may need to be able to approximate the sum of the series in the expression of $\Gamma(s, t)$. Such an example is the Cramér-von Mises test statistic [1], which is the $L^{2}[0,1]$ norm of the empirical process of $U[0,1]$ random variables. So, with dependent variables, we have an asymptotic distribution for this test statistic, but we cannot characterize it completely because we do not know the covariance function $\Gamma(s, t)$. The present article intends to be a first step towards this goal, establishing conditions under which we may find estimates of the limit covariance function.

We will be interested in the case of associated random variables, a dependence concept introduced by Esary et al. [2] which we recall here. The random variables $X_{n}, n \geq 1$, are associated if

$$
\operatorname{Cov}\left(f\left(X_{1}, \ldots, X_{n}\right), g\left(X_{1}, \ldots, X_{n}\right)\right) \geq 0
$$

for any $n \in \mathbb{N}$ and any real-valued coordinate-wise increasing functions $f$ and $g$ for which the covariance above exists. As far as convergence in distribution is concerned, this dependence is characterized through its covariance structure [3]. The best-known results proving the convergence of the empirical process, with assumptions on the decrease rate of the covariances, were obtained by Louhichi [4] and Oliveira and Suquet [5,6] in the $D[0,1], L^{2}[0,1]$ and $L^{p}[0,1]$, respectively.

Given the strict stationarity of the sequence $X_{n}, n \geq 1$, we denote the distribution function of the random vector $\left(X_{1}, X_{k+1}\right)$ by $F_{k}$. Under the assumption of association, Henriques and Oliveira [7] studied the histogram estimator for $F_{k}(s, t)$, namely,

$$
\widehat{F}_{k, n}(s, t)=\frac{1}{n-k} \sum_{i=1}^{n-k}\left(I_{(-\infty, s]}\left(X_{i}\right) I_{(-\infty, t]}\left(X_{i+k}\right)\right) .
$$

The strong consistency of this estimator follows if $\lim _{n \rightarrow \infty}(1 / n) \sum_{j=1}^{n} \operatorname{Cov}^{1 / 3}\left(X_{1}, X_{j}\right)=0$, but no rates were provided. In the present article we prove an exponential inequality, Theorem 2.5 , from which a uniform almost sure convergence rate for $\widehat{F}_{k, n}$ is derived.

For the estimation of the terms $\varphi_{k}(s, t)$ with $k \in \mathbb{N}$ fixed, we consider the estimator

$$
\widehat{\varphi}_{k, n}(s, t)=\widehat{F}_{k, n}(s, t)-\widehat{F}_{n}(s) \widehat{F}_{n}(t),
$$

where $\widehat{F}_{n}$ is the empirical distribution function, defined by $\widehat{F}_{n}(s)=(1 / n) \sum_{j=1}^{n} I_{(-\infty, s]}\left(X_{j}\right)$. The infinite sum in the expression of $\Gamma(s, t)$ is estimated by

$$
\sum_{k=1}^{q_{n}} \widehat{\varphi}_{k, n}(s, t)
$$

where $q_{n} \rightarrow+\infty$ such that $q_{n} / n \rightarrow 0$. Finally, an estimator of $\Gamma(s, t)$ is naturally given by

$$
\widehat{\Gamma}_{n}(s, t)=\widehat{F}_{n}(s \wedge t)-\widehat{F}_{n}(s) \widehat{F}_{n}(t)+\sum_{k=1}^{q_{n}}\left(\widehat{\varphi}_{k, n}(s, t)+\widehat{\varphi}_{k, n}(t, s)\right) .
$$


For easier reference, we now present the assumptions to be considered throughout the text.

(S1) $X_{n}, n \geq 1$, is an associated and strictly stationary sequence of random variables having density function bounded by $B_{0}$; let $B_{1}=2 \max \left(2 / \pi^{2}, 45 B_{0}\right)$.

To derive the exponential inequality, we will find a condition on the decrease rate of $\operatorname{Cov}\left(X_{1}, X_{n}\right)$, which is not very explicit. In order to present a simple condition on the decrease rate of $\operatorname{Cov}\left(X_{1}, X_{n}\right)$, we have to assume some particular behaviour on this sequence.

(G) Suppose that there exist $a_{0}>0$ and $a>1$ such that $\operatorname{Cov}\left(X_{1}, X_{n+1}\right)=a_{0} a^{-n}$.

As previously mentioned, in section 2 we will establish an exponential inequality which is the basis for the proofs of the convergence results of section 3. In this last section, we will derive convergence rates for the uniform strong convergence of the estimators studied here. In this article, $C$ denotes a generic positive constant which may take different values in each appearance; this will not be mentioned again in order to avoid unnecessary repetition.

\section{Notation and preliminary results}

In this section, we introduce notations and state some preliminary results needed for the proofs of the results of the next section. First, let $t_{n}$ be a sequence of positive integers such that $t_{n} \rightarrow \infty$. For each $n \in \mathbb{N}$ and each $i=1, \ldots, t_{n}$, put $x_{n, i}=Q\left(i / t_{n}\right)$, where $Q$ is the quantile function of $F$. In order to simplify the expressions that will follow, we define, for $n, k \in \mathbb{N}$,

$$
D_{n, k}=\sup _{s, t \in \mathbb{R}}\left|\widehat{F}_{k, n}(s, t)-F_{k}(s, t)\right| \quad \text { and } \quad D_{n, k}^{*}=\max _{i, j=1, \ldots, t_{n}}\left|\widehat{F}_{k, n}\left(x_{n, i}, x_{n, j}\right)-F_{k}\left(x_{n, i}, x_{n, j}\right)\right| \text {. }
$$

The following lemma will be used to obtain an exponential inequality for $D_{n, k}$. The proof of this lemma is contained in the proof of Theorem 2 of Henriques and Oliveira [7].

LEMMA 2.1 If the sequence $X_{n}, n \geq 1$, satisfies (S1), then, for each $n \in \mathbb{N}$ and each $k \in \mathbb{N}, D_{n, k} \leq D_{n, k}^{*}+2 / t_{n}$, almost surely.

Now, we introduce some more notation to be used in the sequel. Let $p_{n}$ be a sequence of positive integers such that $p_{n} \rightarrow+\infty$. For each $k \in \mathbb{N}$ and $n \in \mathbb{N}$ large enough, let $r_{k, n}$ be the largest integer such that $r_{k, n} \leq(n-k) /\left(2 p_{n}\right)$. Now, divide the set $\{1, \ldots, n-k\}$ into pairs of subsets, each one containing $p_{n}$ elements. The number of such subsets with $p_{n}$ elements is $2 r_{k, n}$. The last subset in the partition of $\{1, \ldots, n-k\}$ will have $n-k-2 r_{k, n} p_{n}<2 p_{n}$ elements. We will suppose that for each $k \in \mathbb{N}, r_{k, n} \rightarrow+\infty$. The choice of these sequences is crucial for the proof of the exponential inequality. These sequences must be well tuned with the behaviour of the covariance structure of the variables $X_{n}, n \geq 1$.

For the sequences just defined, we have, for each $k \in \mathbb{N}$ and $n \in \mathbb{N}$ large enough, $1 \leq(n-k) /\left(2 r_{k, n} p_{n}\right) \leq\left(2 p_{n}+2 r_{k, n} p_{n}\right) /\left(2 r_{k, n} p_{n}\right)=\left(1+r_{k, n}\right) / r_{k, n}$, so that for each $k \in \mathbb{N}$,

$$
\frac{n-k}{2 r_{k, n} p_{n}} \longrightarrow 1 \text {. }
$$

Note also that, for each $n \in \mathbb{N}$, the set $\{1, \ldots, n-1\}$ has more $p_{n}-1$ elements than $\left\{1, \ldots, n-p_{n}\right\}$. So, the partition of the first set will have at most two more subsets than the partition of the last one. For fixed $k \in \mathbb{N}$, we will eventually have $k<p_{n}$, and this means that, for each $k \in \mathbb{N}$ and $n \in \mathbb{N}$ large enough, we have $r_{k, n}=r_{1, n}$ or $r_{k, n}=r_{1, n}-1$. 
Let us define the sets $E_{i}=\left\{2(i-1) p_{n}+1, \ldots,(2 i-1) p_{n}\right\}, O_{i}=\left\{(2 i-1) p_{n}+\right.$ $\left.1, \ldots, 2 i p_{n}\right\}$, for each $i=1, \ldots, r_{k, n}$, and $R=\left\{2 r_{k, n} p_{n}+1, \ldots, n-k\right\}$.

For each $n, k \in \mathbb{N}$, and fixed $s, t \in \mathbb{R}$, define the random variables

$$
W_{k, n}=I_{(-\infty, s]}\left(X_{n}\right) I_{(-\infty, t]}\left(X_{k+n}\right)-F_{k}(s, t) .
$$

Note that the random variables $W_{k, n}, n \geq 1$, are bounded by 1 and $E\left(W_{k, n}\right)=0$. Additionally, as the sequence $X_{n}, n \geq 1$, is associated and strictly stationary and the $W_{k, n}$ are decreasing functions of the $X_{n}$, the sequence $W_{k, n}, n \geq 1$, is also associated and strictly stationary.

Ioannides and Roussas [8] were the first authors to prove an exponential inequality for bounded associated variables. They used an approach based on the decomposition of a sum of variables into blocks, which we also follow here. The need for blocking arises from the fact that it does not seem possible to find convenient control on the moment generating function of the sum of associated variables. The blocking decomposition enables an approximation to independence when the blocks are sufficiently far apart. In this article, the same technique will be used, although the approximation to independence is achieved here in a different way. In Ioannides and Roussas [8], a recursive argument was used, which needed some technical manipulations, whereas we use the following result here.

LEMMA 2.2 [9] Let $Y_{1}, Y_{2}, \ldots, Y_{n}$ be associated random variables that are bounded by $a$ constant $M$. Then, for any $\theta>0$,

$$
\left|E\left(\mathrm{e}^{\theta \sum_{i=1}^{n} Y_{i}}\right)-\prod_{i=1}^{n} E\left(\mathrm{e}^{\theta Y_{i}}\right)\right| \leq \theta^{2} \mathrm{e}^{n \theta M} \sum_{1 \leq i<j \leq n} \operatorname{Cov}\left(Y_{i}, Y_{j}\right) .
$$

To obtain the exponential inequality, the sum in $\widehat{F}_{k, n}(s, t)-F_{k}(s, t)=(1 /(n-k)) \sum_{i=1}^{n-k}$ $W_{k, i}$ is decomposed into three parts. To do this, define the random variables

$$
U_{k, i}=\sum_{j \in E_{i}} W_{k, j}, \quad V_{k, i}=\sum_{j \in O_{i}} W_{k, j}, \quad i=1, \ldots, r_{k, n} \quad \text { and } \quad Z_{k, n}=\sum_{j \in R} W_{k, j} .
$$

Now, set

$$
\bar{U}_{k, n}=\frac{1}{n-k} \sum_{i=1}^{r_{k, n}} U_{k, i}, \quad \bar{V}_{k, n}=\frac{1}{n-k} \sum_{i=1}^{r_{k, n}} V_{k, i} \quad \text { and } \quad \bar{Z}_{k, n}=\frac{1}{n-k} Z_{k, n},
$$

so that

$$
\widehat{F}_{k, n}(s, t)-F_{k}(s, t)=\bar{U}_{k, n}+\bar{V}_{k, n}+\bar{Z}_{k, n} .
$$

Using Lemma 2.2, we will now prove an exponential inequality for the variables $\bar{U}_{k, n}$, which also holds for $\bar{V}_{k, n}$, and finally, we will show that the remainder term, $\bar{Z}_{k, n}$, is negligible.

We prove a first exponential inequality assuming a general hypothesis on the covariances. As it will be seen in the proof of Theorem 2.5, this assumption is satisfied under $(G)$.

LEMMA 2.3 Let $u_{n}, n \geq 1$, be some sequence of positive numbers. Suppose that (S1) is satisfied, $C(k)=\operatorname{Cov}\left(X_{1}, X_{k+1}\right)$ is non-increasing as $k \rightarrow \infty$ and there exists a constant $C_{1}>0$ such that

$$
C\left(p_{n}-k\right) \leq C_{1} \exp \left(-18 r_{k, n} u_{n}\right)
$$

Then,

$$
P\left(\left|\bar{U}_{k, n}\right| \geq u_{n}\right) \leq C_{2} \exp \left(-2 r_{k, n} u_{n}^{2}\right)
$$

where $C_{2}=2+B_{1} C_{1}^{1 / 3}$. 
Proof The variables $U_{k, 1}, U_{k, 2}, \ldots, U_{k, r_{k, n}}$, being the sum of associated random variables, are associated. Additionally, $\left|U_{k, i}\right| \leq p_{n}$ for every $i=1, \ldots, r_{k, n}$. Then, we may apply Lemma 2.2 to obtain that, given $\lambda>0$,

$$
E\left(\mathrm{e}^{\lambda \bar{U}_{k, n}}\right) \leq \prod_{i=1}^{r_{k, n}} E\left(\mathrm{e}^{\lambda /(n-k) U_{k, i}}\right)+\frac{\lambda^{2}}{(n-k)^{2}} \exp \left(\frac{r_{k, n} p_{n} \lambda}{n-k}\right) \sum_{1 \leq i<j \leq r_{k, n}} \operatorname{Cov}\left(U_{k, i}, U_{k, j}\right) .
$$

As the density of the variables is supposed to be bounded by $B_{0}$, it follows from corollary to Theorem 1 in Sadikova [10] and relation (21) in Newman [11] (see Lemma 2.6 in [12] for details) that

$$
\operatorname{Cov}\left(I_{(-\infty, s]}\left(X_{i}\right), I_{(-\infty, t]}\left(X_{j}\right)\right) \leq B_{1} \operatorname{Cov}^{1 / 3}\left(X_{i}, X_{j}\right), \quad s, t \in \mathbb{R},
$$

where $B_{1}$ has been defined earlier. Now, applying a classical inequality by Lebowitz [13] and taking account of inequality (5), we find

$$
\operatorname{Cov}\left(W_{k, l}, W_{k, m}\right) \leq B_{1}\left[2 \operatorname{Cov}^{1 / 3}\left(X_{l}, X_{m}\right)+\operatorname{Cov}^{1 / 3}\left(X_{l}, X_{k+m}\right)+\operatorname{Cov}^{1 / 3}\left(X_{k+l}, X_{m}\right)\right] .
$$

So, as $C(k)$ is non-increasing, it follows that for $m \in E_{i}$ and $l \in E_{j}$, with $i \neq j$, $\operatorname{Cov}\left(W_{k, l}, W_{k, m}\right) \leq 4 B_{1} C^{1 / 3}\left(p_{n}-k\right)$. Therefore

$$
\begin{aligned}
\sum_{1 \leq i<j \leq r_{k, n}} \operatorname{Cov}\left(U_{k, i}, U_{k, j}\right) & \leq \sum_{i=1}^{r_{k, n}-1} \sum_{j=i+1}^{r_{k, n}} \sum_{l \in E_{i}} \sum_{m \in E_{j}} 4 B_{1} C^{1 / 3}\left(p_{n}-k\right) \\
& =2 r_{k, n}\left(r_{k, n}-1\right) p_{n}^{2} B_{1} C^{1 / 3}\left(p_{n}-k\right) .
\end{aligned}
$$

By construction of the sequences $r_{k, n}$ and $p_{n}$, we have $2 r_{k, n} p_{n} \leq n-k$. It then follows that

$$
\frac{2 p_{n}^{2} r_{k, n}}{(n-k)^{2}} \leq \frac{1}{2 r_{k, n}} \quad \text { and } \quad \frac{p_{n} r_{k, n}}{n-k} \leq \frac{1}{2}
$$

From the preceding considerations, inequality (4) then becomes

$$
\begin{aligned}
E\left(\mathrm{e}^{\lambda \bar{U}_{k, n}}\right) & \leq \prod_{i=1}^{r_{k, n}} E\left(\mathrm{e}^{\lambda /(n-k) U_{k, i}}\right)+\mathrm{e}^{\lambda / 2} \lambda^{2} \frac{r_{k, n}-1}{2 r_{k, n}} B_{1} C^{1 / 3}\left(p_{n}-k\right) \\
& \leq \exp \left(\frac{\lambda^{2}}{8 r_{k, n}}\right)+\frac{B_{1}}{2} \mathrm{e}^{3 \lambda / 2} C^{1 / 3}\left(p_{n}-k\right) ;
\end{aligned}
$$

noticing that for $x>0, x^{2} \leq \mathrm{e}^{x}$, and applying Lemma 1 in Devroye [14]. Then, by the Markov inequality [15], we obtain

$$
P\left(\bar{U}_{k, n} \geq u_{n}\right) \leq \exp \left(-\lambda u_{n}+\frac{\lambda^{2}}{8 r_{k, n}}\right)+\frac{B_{1}}{2} \mathrm{e}^{3 \lambda / 2-\lambda u_{n}} C^{1 / 3}\left(p_{n}-k\right) .
$$

In order to minimize the first term on the right of this previous inequality, we choose $\lambda=4 r_{k, n} u_{n}$. We then find that

$$
P\left(\bar{U}_{k, n} \geq u_{n}\right) \leq\left(1+\frac{1}{2} B_{1} C_{1}^{1 / 3}\right) \exp \left(-2 r_{k, n} u_{n}^{2}\right),
$$

using condition (3). As the variables $-U_{k, i}, i=1, \ldots, r_{k, n}$, have the same properties as the variables $U_{k, i}, i=1, \ldots, r_{k, n}$, this inequality also holds for $-\bar{U}_{k, n}$, which completes the proof. 
The next theorem states the conditions under which we may consider the sum in the last block of decomposition (2) negligible.

LEMMA 2.4 Let $k \in \mathbb{N}$ be fixed and $u_{n}$ be a sequence of positive numbers such that $r_{1, n} u_{n} \rightarrow+\infty$. Then, under (S1), $P\left(\left|\bar{Z}_{k, n}\right| \geq u_{n}\right)=0$, for every sufficiently large $n$.

Proof Note that $\bar{Z}_{k, n}$, being the sum of $(n-k)-2 r_{k, n} p_{n}<2 p_{n}$ variables, which are bounded by 1 , satisfies $\left|\bar{Z}_{k, n}\right| \leq 2 p_{n} /(n-k)$. We then have, for every sufficiently large $n, P\left(\left|\bar{Z}_{k, n}\right| \geq u_{n}\right) \leq P\left(2 p_{n} /(n-k) \geq u_{n}\right)=P\left(2 p_{n} /\left(u_{n}(n-k)\right) \geq 1\right)=0$, on account of convergence (1) and the assumption $r_{1, n} u_{n} \rightarrow+\infty$.

The next theorem establishes an exponential probability inequality for the estimator $\widehat{F}_{k, n}$, which will be used to obtain almost sure convergence rates.

THEOREM 2.5 Let $k \in \mathbb{N}$ be fixed and $u_{n}$ be a sequence of positive numbers such that $u_{n} \rightarrow 0$ and $n u_{n} \rightarrow+\infty$. Also, let $t_{n}$ be a sequence of positive integers such that $t_{n} \rightarrow+\infty$ and $u_{n} t_{n} \rightarrow$ $+\infty$. Further assume that the assumptions $(\mathrm{S} 1)$ and $(\mathrm{G})$ hold and let $b \in\left(0,\left(\log ^{1 / 2} a\right) / 108\right)$. Then, for every sufficiently large $n$,

$$
P\left(\sup _{s, t, \in \mathbb{R}}\left|\widehat{F}_{k, n}(s, t)-F_{k}(s, t)\right| \geq u_{n}\right) \leq C_{3} t_{n}^{2} \exp \left(-b n^{1 / 2} u_{n}^{3 / 2}\right),
$$

where $C_{3}$ is a constant independent of $n$.

Proof Let $r_{1, n}=\left[18 b n^{1 / 2} / u_{n}^{1 / 2}\right]$, where $[x]$ denotes the integer part of $x$. First, we will check that condition (3) is satisfied. Under $(\mathrm{G})$, this condition takes the form $a^{-\left(p_{n}-k\right)} \leq$ $C \exp \left(-18 r_{k, n} u_{n}\right)$. Given that $r_{k, n} \leq r_{1, n}$, this inequality follows from $-\left(p_{n}-k\right) \log a \leq C-$ $18 r_{1, n} u_{n}$. Because of convergence (1), we can write $p_{n}=x_{n} n / r_{1, n}$, for some $0<x_{n} \rightarrow 1 / 2$, so that this inequality becomes $-\log a\left(x_{n} n / r_{1, n}-k\right)+18 r_{1, n} u_{n} \leq C$. Rewriting this as

$$
n^{1 / 2} u_{n}^{1 / 2}\left(-\log a x_{n} \frac{n^{1 / 2}}{r_{1, n} u_{n}^{1 / 2}}+\frac{k \log a}{n^{1 / 2} u_{n}^{1 / 2}}+18 \frac{r_{1, n} u_{n}^{1 / 2}}{n^{1 / 2}}\right) \leq C
$$

and remembering that $r_{1, n} u_{n}^{1 / 2} / n^{1 / 2} \rightarrow 18 b$, it becomes evident that, given the conditions of this Theorem, this inequality is eventually verified. Moreover, it is easy to check that $r_{1, n} u_{n} \rightarrow+\infty$, so the result of Lemma 2.4 holds.

Fix $s, t \in \mathbb{R}$. Taking account of decomposition (2), we obtain, from Lemma 2.3 applied to $\bar{U}_{k, n}$ and to $\bar{V}_{k, n}$, and Lemma 2.4 , for every sufficiently large $n$,

$$
\begin{aligned}
& P\left(\left|\widehat{F}_{k, n}(s, t)-F_{k}(s, t)\right| \geq u_{n}\right) \\
& \quad \leq P\left(\left|\bar{U}_{k, n}\right| \geq \frac{u_{n}}{3}\right)+P\left(\left|\bar{V}_{k, n}\right| \geq \frac{u_{n}}{3}\right)+P\left(\left|\bar{Z}_{k, n}\right| \geq \frac{u_{n}}{3}\right) \\
& \quad \leq C \exp \left(-\frac{2}{9} r_{k, n} u_{n}^{2}\right) \\
& \quad \leq C \exp \left(-\frac{2}{9}\left(r_{1, n}-1\right) u_{n}^{2}\right) .
\end{aligned}
$$


Using Lemma 2.1 and remembering that $u_{n} t_{n} \rightarrow+\infty$, we obtain, for every sufficiently large $n$,

$$
\begin{aligned}
P\left(D_{n, k} \geq u_{n}\right) & \leq P\left(D_{n, k}^{*} \geq \frac{u_{n}}{2}\right)+P\left(\frac{2}{t_{n}} \geq \frac{u_{n}}{2}\right) \\
& \leq t_{n}^{2} \max _{i, j=1, \ldots, t_{n}} P\left(\left|\widehat{F}_{k, n}\left(x_{n, i}, x_{n, j}\right)-F_{k}\left(x_{n, i}, x_{n, j}\right)\right| \geq \frac{u_{n}}{2}\right) .
\end{aligned}
$$

Finally, applying inequality (8), it follows, for every sufficiently large $n$,

$$
P\left(D_{n, k} \geq u_{n}\right) \leq C t_{n}^{2} \exp \left(-\frac{1}{18}\left(r_{1, n}-1\right) u_{n}^{2}\right) \leq C t_{n}^{2} \exp \left(-b n^{1 / 2} u_{n}^{3 / 2}\right)
$$

\section{Convergence and rates}

We are now in a position to derive uniform strong convergence rates for the estimators considered in this paper. We begin with the estimators $\widehat{F}_{k, n}(s, t)$ and $\widehat{\varphi}_{k, n}(s, t)$.

THEOREM 3.1 Let $k \in \mathbb{N}$ be fixed. Suppose that (S1) and (G) are satisfied. Then $\sup _{s, t \in \mathbb{R}}\left|\widehat{F}_{k, n}(s, t)-F_{k}(s, t)\right| \rightarrow 0$ almost surely with rate of order $n^{-1 / 3} \log ^{2 / 3} n$.

Proof Choose $\alpha>5 / 3, b \in\left(0, \log ^{1 / 2} a / 108\right)$ and $t_{n}=\left[n^{1 / 3} / \log ^{1 / 6} n\right]$. Also, let $u_{n}=\left(\frac{\alpha}{b}\right)^{2 / 3} \log ^{2 / 3} n / n^{1 / 3}$, so that $\exp \left(-b n^{1 / 2} u_{n}^{3 / 2}\right)=n^{-\alpha}$. As it is easily verified, these sequences satisfy all the conditions of Theorem 2.5 , from which we have, for every sufficiently large $n$,

$$
P\left(\sup _{s, t \in \mathbb{R}}\left|\widehat{F}_{k, n}(s, t)-F_{k}(s, t)\right| \geq u_{n}\right) \leq C t_{n}^{2} \exp \left(-b n^{1 / 2} u_{n}^{3 / 2}\right) \leq C n^{2 / 3-\alpha} \log ^{-1 / 3} n .
$$

It is clear that the right-hand side of this inequality defines a convergent series, as $\alpha>5 / 3$. The result follows now from the Borel-Cantelli lemma [16].

Note that under (S1) and $(\mathrm{G})$, setting $k=0$ and $s=t$, it follows from Theorem 3.1 that

$$
\sup _{s \in \mathbb{R}}\left|\widehat{F}_{n}(s)-F(s)\right|=O\left(n^{-1 / 3} \log ^{2 / 3} n\right) \quad \text { a.s. }
$$

Moreover, as $\left|F(s) F(t)-\widehat{F}_{n}(s) \widehat{F}_{n}(t)\right|=F(s)\left|F(t)-\widehat{F}_{n}(t)\right|+\widehat{F}_{n}(t)\left|F(s)-\widehat{F}_{n}(s)\right| \leq$ $\left|F(t)-\widehat{F}_{n}(t)\right|+\left|F(s)-\widehat{F}_{n}(s)\right|$, Theorem 3.1 also implies that under (S1) and (G),

$$
\sup _{s, t \in \mathbb{R}}\left|F(s) F(t)-\widehat{F}_{n}(s) \widehat{F}_{n}(t)\right|=O\left(n^{-1 / 3} \log ^{2 / 3} n\right) \quad \text { a.s. }
$$

The previous remarks serve to prove a characterization of the almost sure convergence of the estimator $\widehat{\varphi}_{k, n}$ for the general term of the series in the expansion of $\Gamma(\cdot, \cdot)$.

TheOREM 3.2 Let $k \in \mathbb{N}$ be fixed. Suppose that (S1) and (G) are satisfied, then $\sup _{s, t \in \mathbb{R}}\left|\widehat{\varphi}_{k, n}(s, t)-\varphi_{k}(s, t)\right|=O\left(n^{-1 / 3} \log ^{2 / 3} n\right)$, almost surely. 
Proof Write

$$
\sup _{s, t \in \mathbb{R}}\left|\widehat{\varphi}_{k, n}(s, t)-\varphi_{k}(s, t)\right| \leq \sup _{s, t \in \mathbb{R}}\left|\widehat{F}_{k, n}(s, t)-F_{k}(s, t)\right|+\sup _{s, t \in \mathbb{R}}\left|F(s) F(t)-\widehat{F}_{n}(s) \widehat{F}_{n}(t)\right| .
$$

Thus, the theorem follows from Theorem 3.1, together with relation (10).

The uniform strong consistency of the estimators for the infinite sum $\sum_{k=1}^{\infty} \varphi_{k}(s, t)$ and for $\Gamma(s, t)$ is established in the next result.

THEOREM 3.3 Let assumptions (S1) and $(\mathrm{G})$ hold. If $q_{n}=O\left(n^{1 / 3} \log ^{-\delta} n\right)$, for some $\delta>2 / 3$, then

$$
\begin{array}{r}
\sup _{s, t \in \mathbb{R}}\left|\sum_{k=1}^{q_{n}} \widehat{\varphi}_{k, n}(s, t)-\sum_{k=1}^{\infty} \varphi_{k}(s, t)\right| \longrightarrow 0 \quad \text { a.s., } \\
\sup _{s, t \in \mathbb{R}}\left|\widehat{\Gamma}_{n}(s, t)-\Gamma(s, t)\right| \longrightarrow 0 \text { a.s. }
\end{array}
$$

Proof Choose $\alpha>2, b \in\left(0, \log ^{1 / 2} a / 108\right)$ and $t_{n}$ and $u_{n}$ as in the proof of Theorem 3.1, for which all conditions of Theorem 2.5 are verified. Here, it is worth noting that for each $k=1, \ldots, q_{n}$, the constant $C_{3}$ in inequality (6) is independent of $k$. In fact, $C_{3}$ depends on $C$, the generic constant in inequality (7). Although looking at inequality (7) it seems that this constant depends on $k$, we may conclude that, in fact, it does not. To justify this observation, as $q_{n}\left(n u_{n}\right)^{-1 / 2} \rightarrow 0$, we can replace $k$ with $q_{n}$ in inequality (7), making it independent of $k$. Furthermore, there is an $n_{0} \in \mathbb{N}$ such that inequality (6) holds true for all $n \geq n_{0}$ and for all $k=1, \ldots, q_{n}$. This is so because, as $2 p_{n} /\left(u_{n}\left(n-q_{n}\right)\right) \rightarrow 0$, in Lemma 2.4, we can choose $n_{0} \in \mathbb{N}$ such that for every $n \geq n_{0}$ and for every $k=1, \ldots, q_{n}, 2 p_{n} /\left(u_{n}(n-k)\right) \leq$ $2 p_{n} /\left(u_{n}\left(n-q_{n}\right)\right)<1$.

Let $\varepsilon_{n}=q_{n} u_{n}$. From Theorem 2.5 we then have, for every sufficiently large $n$,

$$
\begin{aligned}
& P\left(\sup _{s, t \in \mathbb{R}}\left|\sum_{k=1}^{q_{n}}\left(\widehat{F}_{k, n}(s, t)-F_{k}(s, t)\right)\right| \geq \varepsilon_{n}\right) \\
& \quad \leq \sum_{k=1}^{q_{n}} \mathrm{P}\left(\sup _{s, t \in \mathbb{R}}\left|\widehat{F}_{k, n}(s, t)-F_{k}(s, t)\right| \geq \frac{\varepsilon_{n}}{q_{n}}\right) \\
& \leq C q_{n} t_{n}^{2} \exp \left(-b n^{1 / 2} u_{n}^{3 / 2}\right) \leq C n^{-(\alpha-1)} \log ^{-(\delta+1 / 3)} .
\end{aligned}
$$

As $\alpha-1>1$, the last sequence above defines a convergent series. As it is easily verified $\varepsilon_{n} \rightarrow 0$, so the Borel-Cantelli lemma yields $\sup _{s, t \in \mathbb{R}}\left|\sum_{k=1}^{q_{n}}\left(\widehat{F}_{k, n}(s, t)-F_{k}(s, t)\right)\right| \rightarrow 0$ almost surely. Now, convergence (11) follows from

$$
\begin{aligned}
& \sup _{s, t \in \mathbb{R}}\left|\sum_{k=1}^{q_{n}} \widehat{\varphi}_{k, n}(s, t)-\sum_{k=1}^{\infty} \varphi_{k}(s, t)\right| \\
& \leq \sup _{s, t \in \mathbb{R}}\left|\sum_{k=1}^{q_{n}}\left(\widehat{F}_{k, n}(s, t)-F_{k}(s, t)\right)\right| \\
& \quad+q_{n} \sup _{s, t \in \mathbb{R}}\left|F(s) F(t)-F_{n}(s) F_{n}(t)\right|+\sup _{s, t \in \mathbb{R}}\left|\sum_{k=q_{n}+1}^{\infty} \varphi_{k}(s, t)\right| .
\end{aligned}
$$

In fact, as we have just established, the first term on the right-hand side above converges almost surely to zero. The convergence of the second term follows from relation (10), as 
$q_{n} \leq C n^{1 / 3} \log ^{-\delta} n$, where $\delta>2 / 3$. Moreover, as mentioned before (see inequality (5)), $\varphi_{k}(s, t) \leq B_{1} \operatorname{Cov}^{1 / 3}\left(X_{1}, X_{k+1}\right)$, for every $s, t \in \mathbb{R}$, and as (G) implies that $\sum_{k} \operatorname{Cov}^{1 / 3}\left(X_{1}, X_{k+1}\right)<\infty$, it follows that the third term of the upper bound above also converges to zero as $q_{n} \rightarrow+\infty$.

To prove convergence (12), we write

$$
\begin{aligned}
\sup _{s, t \in \mathbb{R}} & \left|\widehat{\Gamma}_{n}(s, t)-\Gamma(s, t)\right| \\
\leq & \sup _{s, t \in \mathbb{R}}\left|\widehat{F}_{n}(s \wedge t)-F(s \wedge t)\right|+\sup _{s, t \in \mathbb{R}}\left|F(s) F(t)-\widehat{F}_{n}(s) \widehat{F}_{n}(t)\right| \\
& \quad+\sup _{s, t \in \mathbb{R}}\left|\sum_{k=1}^{q_{n}} \widehat{\varphi}_{k, n}(s, t)-\sum_{k=1}^{\infty} \varphi_{k}(s, t)\right|+\sup _{s, t \in \mathbb{R}}\left|\sum_{k=1}^{q_{n}} \widehat{\varphi}_{k, n}(t, s)-\sum_{k=1}^{\infty} \varphi_{k}(t, s)\right| .
\end{aligned}
$$

The almost sure convergence to zero of the first and second terms of the right-hand side above follows, respectively, from relations (9) and (10). Finally, the third and fourth terms converge to zero according to convergence (11).

The previous result does not identify a convergence rate. An optimization of the choice of the sequence $q_{n}$ gives the best convergence rates that follow from the exponential inequality of Theorem 2.5 .

Theorem 3.4 Suppose (S1) and (G) hold. If $q_{n}=[\log n]$ and $\beta \leq \min \{1 / 3, \log a / 3\}$, then

$$
\sup _{s, t \in \mathbb{R}}\left|\sum_{k=1}^{q_{n}} \widehat{\varphi}_{k, n}(s, t)-\sum_{k=1}^{\infty} \varphi_{k}(s, t)\right|=O\left(n^{-\beta} \log ^{5 / 3} n\right) \quad \text { a.s., }
$$

and

$$
\sup _{s, t \in \mathbb{R}}\left|\widehat{\Gamma}_{n}(s, t)-\Gamma(s, t)\right|=O\left(n^{-\beta} \log ^{5 / 3} n\right) \quad \text { a.s. }
$$

Proof Choose $\alpha>5 / 3, b \in\left(0, \log ^{1 / 2} a / 108\right)$ and $t_{n}$ and $u_{n}$ as in the proof of Theorem 3.1, for which all conditions of Theorem 2.5 are verified. We again note that for each $k=1, \ldots, q_{n}$, the constant $C_{3}$ in inequality (6) is independent of $k$. To verify this, just follow the arguments used in the proof of Theorem 3.3, noting that we have again $q_{n}\left(n u_{n}\right)^{-1 / 2} \rightarrow 0$. Also, there is an $n_{0} \in \mathbb{N}$ such that inequality (6) holds true for all $n \geq n_{0}$ and for all $k=1, \ldots, q_{n}$.

Arguing as in the proof of the previous theorem we then have, for $\varepsilon_{n}=q_{n} u_{n}$,

$$
P\left(\sup _{s, t \in \mathbb{R}}\left|\sum_{k=1}^{q_{n}}\left(\widehat{F}_{k, n}(s, t)-F_{k}(s, t)\right)\right| \geq \varepsilon_{n}\right) \leq C n^{-(\alpha-2 / 3)} \log ^{2 / 3} n,
$$

because $\alpha-2 / 3>1$, this upper bound defines a convergent series. As $\varepsilon_{n}=q_{n} u_{n} \sim$ $n^{-1 / 3} \log ^{5 / 3} n$, we then have $\sup _{s, t \in \mathbb{R}}\left|\sum_{k=1}^{q_{n}}\left[\widehat{F}_{k, n}(s, t)-F_{k}(s, t)\right]\right|=O\left(n^{-\beta} \log ^{5 / 3} n\right)$, almost surely. Now, consider the decomposition (13). The rate for the first term on its righthand side has just been established. According to relation (10) and $\beta \leq 1 / 3$, the second term is of the same order. To prove relation (15), we still have to check that also the third term is $O\left(n^{-\beta} \log ^{5 / 3} n\right)$. For this, just apply inequality (5) to obtain

$$
0 \leq \sup _{s, t \in \mathbb{R}} \sum_{k=q_{n}+1}^{\infty} \varphi_{k}(s, t) \leq B_{1} \sum_{k=q_{n}+1}^{\infty} \operatorname{Cov}^{1 / 3}\left(X_{1}, X_{k+1}\right) \leq C \sum_{k=q_{n}+1}^{\infty} a^{-k / 3} \leq \mathrm{Ca}^{-q_{n} / 3},
$$

and notice that $a^{-q_{n} / 3}$ is $O\left(n^{-\beta} \log ^{5 / 3} n\right)$, as $\beta \leq \min \{1 / 3, \log a / 3\}$. 
Finally, to prove relation (16) use decomposition (14) and apply relations (9), (10) and (15).

The last theorem states that under $(\mathrm{G})$ with $a>e$, the estimators for the infinite sum $\sum_{k=1}^{\infty} \varphi_{k}(s, t)$ and for $\Gamma(s, t)$ are uniformly strong consistent with a convergence rate of order $n^{-1 / 3} \log ^{5 / 3} n$.

To conclude, we note that supposing a slightly weaker condition on the decrease rate of the covariances, namely, $\operatorname{Cov}\left(X_{1}, X_{n+1}\right)=a_{0} k^{-\log k}$, with $a_{0}>0$, still enables the derivation of convergence rates for the estimators under consideration. Obviously, the rates under this condition are much slower: of order $\log ^{-1} n$ for $\widehat{\varphi}_{k, n}$ and of order $1 / \log ^{1-\delta} n$ for the estimator of the covariance function, $\widehat{\Gamma}_{n}$, where $\delta \in(0,1)$ may be chosen arbitrarily close to zero.

\section{Acknowledgements}

The authors wish to thank an anonymous referee whose comments helped in improving an earlier version of this article. Carla Henriques was supported by Centro de Matemática da Universidade de Coimbra, Fundação para a Ciência e Tecnologia (FCT), POCTI and PRODEP. Paulo Eduardo Oliveira was supported by Centro de Matemática da Universidade de Coimbra, Fundação para a Ciência e Tecnologia (FCT), POCTI and Fundo Socio Europeu (FSE)PRODEP III.

\section{References}

[1] Shorack, G.R. and Wellner, J.A., 1986, Empirical Processes with Applications to Statistics (New York: Wiley).

[2] Esary, J.D., Proschan, F. and Walkup, D.W., 1967, Association of random variables, with applications. Annals of Mathematical Statistics, 38, 1466-1474.

[3] Newman, C., 1984, Asymptotic independence and limit theorems for positively and negatively dependent random variables. In: Y.L. Tong (Ed.) Inequalities in Statistics and Probability, IMS Lecture Notes-Monograph Series 5, Inst. Math. Statist., Hayward, CA, pp. 127-140.

[4] Louhichi, S., 2000, Weak convergence for empirical processes of associated sequences. Annales de l'Institut Henri Poincaré. Probabilitiés et Statistiques, 36, 547-567.

[5] Oliveira, P.E. and Suquet, C., 1995, $L^{2}[0,1]$ weak convergence of the empirical process for dependent variables. In: A. Antoniadis and G. Oppenheim (Eds.), Lecture Notes in Statistics 103, Wavelets and Statistics. Actes des XV Rencotres Franco-Belges de Statisticiens (Ondelettes et Statistique), pp. 331-344.

[6] Oliveira, P.E. and Suquet, C., 1998, Weak convergence in $L^{p}[0,1]$ of the uniform empirical process under dependence. Statistics and Probability Letters, 39, 363-370.

[7] Henriques, C. and Oliveira, P.E., 2003, Estimation of a two dimensional distribution function under association. Journal of Statistical Planning and Inference, 113, 137-150.

[8] Ioannides, D.A. and Roussas, G.G., 1998, Exponencial inequality for associated random variables. Statistics and Probability Letters, 42, 423-431.

[9] Dewan, I. and Prakasa Rao, B.L.S., 1999, A general method of density estimation for associated random varibles. Journal of Nonparametric Statistics, 10, 405-420.

[10] Sadikova, S.M., 1996, Two-dimensional analogies of an inequality of Esseen with applications to the central limit thorem. Theory of Probability and its Applications, 11, 325-335.

[11] Newman, C.M., 1980, Normal fluctuations and the FKG inequalities. Communications on Mathematical Physics, 74, 119-128.

[12] Roussas, G.G., 1995, Asymptotic normality of a smooth estimate of a random field distribution function under association. Statistics and Probability Letters, 24, 77-90.

[13] Lebowitz, J., 1972, Bounds on the correlations and analycity properties of ferromagnetic Ising spin systems. Communications on Mathematical Physics, 28, 313-321.

[14] Devroye, L., 1991, Exponential inequalities in nonparametric estimation. In: G. Roussas (Ed.), Nonparametric Functional Estimation and Related Topics (Dordrecht: Kluwer Academic Publishers), pp. 31-44.

[15] Loéve, M., 1977, Probability Theory I (New York: Springer-Verlag), p. 160.

[16] Loéve, M., 1977, Probability Theory I (New York: Springer-Verlag), p. 240. 